\title{
Neuro-Immunotherapies: A 30-year Retrospective of an Overwhelming Success and a Brighter Future
}

\author{
Marinos C. Dalakas ${ }^{1,2}$
}

Published online: 18 December 2015

(C) The American Society for Experimental NeuroTherapeutics, Inc. 2015

Serving as Guest Editor of this issue of Neurotherapeutics on immunotherapy in neurologic diseases is a personal retrospective dating back to 30 years ago, and a testimony to the overwhelming success we have witnessed in treating autoimmune neurological disorders. I first served as Guest Editor on Neuroimmunotherapies for Seminars in Neurology in 1994 [1], and for the second time 10 years later, in 2003 [2]. This is my third run on the same subject, now for Neurotherapeutics, in a special issue that highlights the evolution in the field as a true success story with skyrocketing progress in the last 10 years.

My two prior prefaces, as re-read now [1, 2], convey a strong and persistent enthusiasm for the application of new immunotherapies, expressed first with a cautious optimism in 1994 but with increased confidence in 2003, predicting a very bright future that transcends today into a satisfying fulfilling prophecy. In the 1994 preface [1], summarizing the status of the previous decade, I wrote that "immunotherapy is one of the most rewarding approaches in the practice of neurology; it has taught us to apply boldly new drugs or procedures, even for neurologic diseases with a putative autoimmune pathogenesis, but it has also made us cautious and sceptical, for the therapies may cause complications that are worse

Electronic supplementary material The online version of this article (doi:10.1007/s13311-015-0414-2) contains supplementary material, which is available to authorized users.

Marinos C. Dalakas

marinos.dalakas@jefferson.edu

1 Thomas Jefferson University, 901 Walnut Street, Philadelphia, PA, USA

2 Neuroimmunology, National and Kapodistrian University of Athens, Medical School, Athens, Greece than the disease we are treating". How true this view stands today, especially when facing rare catastrophic complications such as progressive multifocal leukoencephalopathy. It is amazing, however, that the only available therapies discussed by all the authors at that time were prednisone, intravenous immunoglobulin (IVIg), plasmapheresis, azathioprine and cyclophosphamide, with IVIg emerging as the rising star, with "beta-interferon as probably having some benefit in multiple sclerosis' [1]. In the ensuing 10 years, there was a remarkable change in scene, as highlighted in the 2004 preface, where I wrote, "the explosion in biotechnology and the developments of new therapeutic agents based on molecular immunology models have been now wisely applied by clinical neurologists. As a result, new drugs have been tested, controlled trials have been conducted and a variety of new agents have been approved. Most importantly, the new therapies are increasingly target-oriented against molecules associated with $\mathrm{T}$ cell activation and transmigration, co-stimulatory molecules, cytokines and adhesion molecules", concluding that "judging from the progress made over the last 10 years and the galloping pace of new discoveries especially in the form of targetspecific monoclonal antibodies, the future of immunotherapies for autoimmune neurological diseases is not what used to be". How true this has proven to be.

Now, 10 years later, we are living the golden era of neuroimmunotherapies, with a success probably surpassing any other field in neurologic therapeutics. Some of my colleagues and experts in the field, who have contributed to this issue, have also witnessed this unprecedented progress that has brought great benefits to our patients. Not only do we have effective agents proven in controlled studies, but we have also learned how to conduct better trials, have identified biomarkers for prognosis and therapeutic monitoring, and have successfully applied therapies against key molecules and pathways associated with tissue damage that effectively target 1) 
T-cell intracellular signaling pathways and molecules associated with antigen presentation; 2) B cells, B-cell trophic factors, and autoantibodies; 3) Fc receptor modulation; 4) complement; 5) cytokines, cytokine receptors, and regulatory $\mathrm{T}$ cells; and 6) molecules involved in cell adhesion and T-cell migration [3]. Consequently, it is really the best of times to serve again as Guest Editor, celebrating these successes together with outstanding colleagues who have done pioneering work in the field, presenting the latest in therapeutics for the major autoimmune neurological diseases.

The progress in the field is not only limited to new therapies, but also to a remarkable identification of new autoimmune neurological diseases, as reflected in the list of topics covered in this issue compared with the two previous ones I was involved in $[1,2]$. Diseases like multiple sclerosis (MS), now covered by 3 different subtypes (relapsing-remitting, primary progressive, and pediatric) with 12 medications on the market, neuromyelitis spectrum disorders, autoimmune painful neuropathic syndromes, autoimmune encephalopathies and hyperexciatability disorders, even neurodegenerative diseases, are a testament to advances in recognizing new autoimmune phenomena in less than 10 years. Progress on targeting molecules on T and B cells, covered now in specific articles, is further testimomy to ongoing target-specific therapies.

The present issue starts with translational science on $\mathrm{T}$ and B cells. Bittner and Wiendl [4] discuss the ways of modifying the immune response to influence disease progression by targeting T-cell-associated transduction molecules, highlighting future molecular anti-T-cell therapies, especially in patients with MS. Alexopoulos et al. [5] discuss the same principles for B cells, including B-cell trophic factors, emphasizing the role of $\mathrm{B}$ cells in neurological diseases and the unprecedented effect the anti-B-cell therapies are expected to play as neuroimmunotherapies in the immediate future. Lünemmn et al. [6] summarize the evidence-based indications for the use of IVIg in neurology, including potential biomarkers of response to therapies, and future strategies on how to replace IVIg with recombinant products to enhance its antiinflammatory properties. Ingwersen et al. [7] provide an upto-date algorithm on how best to treat relapsing-remitting MS using the 12 drugs available on the market, with advice and guidance on how best to proceed with escalation therapy. Shirani et al. [8] identify novel trial designs, discuss the new promising drugs, and offer practical advice on how to manage primary progressive MS. Brenton and Banwell [9] address the present therapies in pediatric MS and highlight the current controversies in diagnosing and treating acute and chronic childhood demyelinating diseases. Kleiter and Gold [10] provide an updated therapeutic guidance on the best means to manage the neuromyelitits optica spectrum disorders, pointing out the evolving new therapies. Léger et al. [11] provide an up-to-date therapeutic scheme for all autoimmune neuropathies, while also addressing recent uncertainties regarding the treatment of chronic inflammatory demyelinating polyneuropathy with nodal antibodies and patients with antimyelin-associated glycoprotein neuropathies. Oaklander [12] tackles the controversial issue of autoimmune painful neuropathies that include small fiber-predominant ganglionitis, complex regional pain syndrome, and distal painful polyneuropathies, providing guidance on how not to miss this potentially treatable subset of autoimmune painful syndromes, and advising on better management. Guptill et al. [13] summarize the present and future translational therapies in myasthenia gravis, while Needham and Mastaglia [14] outline a practical approach to treating the different subtypes of autoimmune inflammatory myopathies as identified today. Gastaldi et al. [15] discuss the continuously evolving group of autoantibody-mediated autoimmune encephalopathies and excitability disorders, providing an update on the best means to diagnose and treat patients with such multifaceted presentations. Kampylafka et al. [16] discuss how best to identify and treat immune neurological conditions encountered in the context of systemic autoimmune diseases, including neurological complications of the monoclonal antibodies and biological agents as used in general medicine. Finally, Valera et al. [17] tackle the difficult but highly exciting topic of immunotherapies targeting neurodegenerative molecules such as amyloid, $\alpha$-synuclein and $p$-tau as future means of treating neurodegenerative diseases.

These successes have not come without pain, disappointments, and rare but catastrophic complications. Neuroimmunotherapy remains an unfinished business. We still have a lot of ground to cover, especially with regard to safer and longer-lasting agents. In MS, more than any other autoimmune neurological disease, we are seeing a gradual shift from injectable to oral agents while anticipating the emergence of newer drugs that promise not only immunomodulation, but also neuroprotection, longer-lasting efficacy, and long relapse-free periods. Given the direction in which the field is going, it will not be surprising if, 10 years from now, we will have agents exerting both immunomodulation and neuroprotection, in a single or combination therapy; effective means of predicting or treating early the very rare but sometimes catastrophic complications we have all witnessed the last few years; and customized choices for drugs best suited to an individual patient with autoimmune neurological disease or even for a specific stage of the disease.

Putting this issue together required considerable effort. The help of Dr. Harry Alexopoulos, a research fellow in my neuroimmunology laboratory at the Medical School of the University of Athens was critical; he played a key role in organizational logistics and scientific reviews, and I am extremely grateful to him. The editorial assistance of Linda Powell, chasing all of us to be on schedule, was essential. Finally, I believe the conception of this topic by the Editor in Chief - Dr. Maral Mouradian - was a really insightful view of 
the unprecedented contribution this field has made to neurology as a whole. With my thanks to all of the above, and most of all to friends and colleagues who contributed such outstanding reviews, I truly believe this issue will serve as a reference text on immunotherapies for all autoimmune neurological disorders for the years to come and as a landmark to judge the progress in the field another 10 years from now.

Compliance with Ethical Standards Required Author Forms Disclosure forms provided by the authors are available with the online version of this article.

\section{References}

1. Dalakas MC (Guest Editor). Neuroimmunotherapy: a practical approach to the treatment of immune-mediated neurologic diseases. Semin Neurol 1994;14(2):95-194.

2. Dalakas MC (Guest Editor). Neuroimmunotherapy. Semin Neurol 2003;23(Vol 2):115-224.

3. Dalakas MC. Future perspectives in target-specific immunotherapies of Myasthenia Gravis. Therap Adv Neurol Disorders 2015;8:316-327.

4. Bittner S, Wiendl H. Neuorimmunotherapies targeting T cells: From pathophysiology to therapeutic applications. Neurotherapeutics 2016.

5. Alexopoulos H, Biba A, Dalakas MC. Anti-B-cell therapies in autoimmune neurological diseases: Rationale and efficacy trials. Neurotherapeutics 2016.
6. Lünemann JD, Quast I, Dalakas MC. Efficacy of intravenous immunoglobulin in neurological diseases. Neurotherapeutics 2016.

7. Ingwersen J, Aktas O, Hartung, H-P. Advances in and algorithms for the treatment of relapsing-remitting multiple sclerosis. Neurotherapeutics 2016.

8. Shirani A, Okuda DT, Stüve O. Therapeutic advances and future prospects in progressive forms of multiple sclerosis. Neurotherapeutics 2016.

9. Brenton, Banwell. Therapeutic approach to the management of pediatric demyelinating disease: Multiple sclerosis and acute disseminated encephalomyelitis. Neurotherapeutics 2016.

10. Kleiter I, Gold R. Present and future therapies in neuromyelitis optica spectrum disorders. Neurotherapeutics 2016.

11. Léger J-M, Guimarães-Costa R, Muntean C. Immunotherapy in peripheral neuropathies. Neurotherapeutics 2016.

12. Oaklander AL. Immunotherapy prospects for painful small-fiber sensory neuropathies and ganglionopathies. Neurotherapeutics 2016.

13. Guptill JT, Soni M, Mariggioli MN. Current treatment, emerging translational therapies, and new therapeutic targets for autoimmune myasthenia gravis. Neurotherapeutics 2016.

14. Needham M, Mastaglia FL. Immunotherapies for immunemediated myopathies: A current perspective. Neurotherapeutics 2016.

15. Gastaldi M, Thouin A, Vincent A. Antibody-mediated autoimmune encephalopaties and immunotherapies. Neurotherapeutics 2016.

16. Kampylafka EI, Alexopoulos H, Dalakas MC, Tzioufas AG. Immunotherapies for neurological manifestations in the context of systemic autoimmunity. Neurotherapeutics 2016.

17. Valera E, Spencer B, Masliah E. Immunotherapeutic approaches in targeting amyloid- $\beta, \alpha$-synuclein, and tau for the treatment of neurodegenerative disorders. Neurotherapeutics 2016. 